
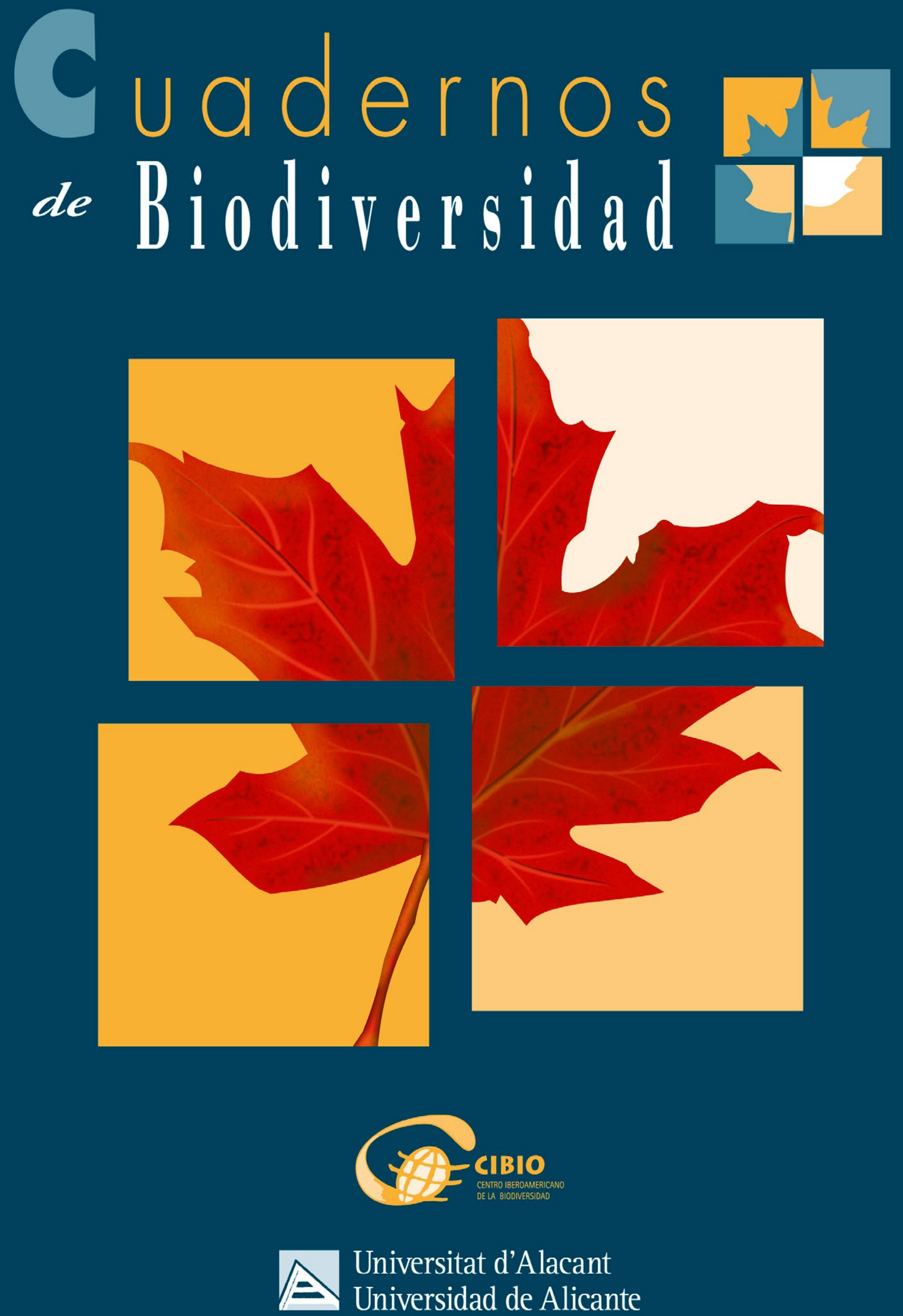


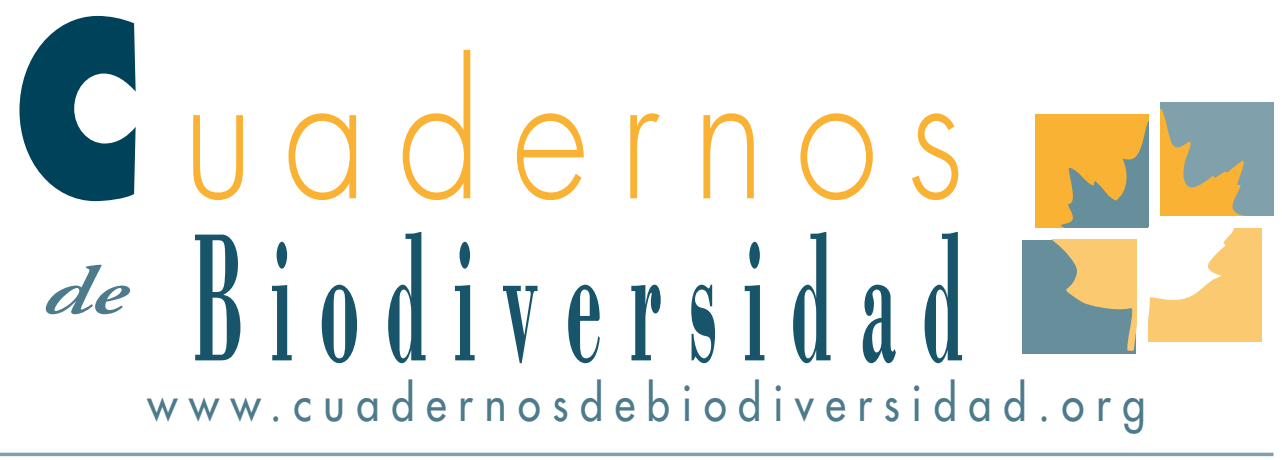

\section{Mariposas diurnas (Lepidoptera) de la Estación Biológica de Torretes (Ilbi,} Alicante)

\section{Diurnal butterflies (Lepidoptera) of the Biological Station of Torretes (Ibi, Alicante)}

\section{F. Castaño ${ }^{1}$, E. Galante ${ }^{2}$}

i Calle Senda del Obispo i 4 (E) Orihuela (Alicante, España)

2 Centro Iberoamericano de la Biodiversidad (CiBIO). Universidad de Alicante. (GALANTE@UA.Es)

\section{RESUMEN}

Las mariposas diurnas, están presentes en todo el mundo y tienen unos requerimientos biológicos que son bien conocidos, por lo que el estudio de sus comunidades nos aporta valiosa información sobre el estado de conservación de los ecosistemas y de los procesos de alteración ambiental. En este estudio se dan a conocer las especies que se encuentran en la Estación Biológica de Torretes, situado en Ibi (Alicante), en la ladera sur del Parque Natural de la Font Roja. Se trata de un espacio municipal de 53 ha cedido en custodia de territorio por el ayuntamiento de Ibi al Centro Iberoamericano de la Biodiversidad (CIBIO) de la Universidad de Alicante. Se han encontrado un total de 32 especies que viven en este espacio natural, lo que supone el $44 \%$ de las especies registradas en el Parque Natural de la Font Roja (Alicante) cuya extensión es de 2.298 ha. Este dato, y la abundancia de las especies registradas, indican la importancia que este espacio tiene como área de conservación entomológica.

Palabras clave: Insectos; Lepidoptera Rhopalocera; Mariposas; Bioindicador; Conservación; Estación Biológica de Torretes. 


\section{ABSTRACT}

The diurnal butterflies, are present all over the world and have biological requirements that are well known, so the study of their communities provide us valuable information on the state of conservation of the ecosystems and the processes of environmental alteration. In this study, species which are found in the Biological Station of Torretes, where is placed in Ibi (Alicante), in the southern hillside of the Natural Park of Font Roja, are made known. It's a municipal area of 53 ha, ceded in custody of territory by the town hall of Ibi to the Ibero-American Center of Biodiversity (CIBIO) of the University of Alicante. A total of 32 species that lives in this natural area had been found, which suppose the $44 \%$ of the species registered in the Natural Park of Font Roja (Alicante) whose extent is 2.298 ha. This data and the abundancy of the registered species, show the importance of this space as a entomological conservation area.

Key words: Insects; Lepidoptera Rhopalocera; Butterflies; Bioindicator; Conservation; Biological Station of Torretes.

\section{INTRODUCCIÓN}

Las mariposas diurnas comprenden unas 18.000 especies que se encuentran distribuidos por todo el mundo, con excepción de las tierras permanentemente heladas (Convey, 2004). Las especies de este grupo, de acuerdo con van Nieukerken et al. (2011) pertenecen en su inmensa mayoría a la superfamilia Papilionoidea Latreille, 1802. Los Papilionoidea ibéricos comprenden 233 especies (Karsholt \& Nieukerken 2017, Monasterio León et al. 2017) lo que supone más del $50 \%$ de las especies que viven en la región mediterránea (Numa et al., 2016), agrupando las familias Papilionidae, Hesperidae, Pieridae, Lycaenidae y Nymphalidae, incluyéndose en esta última familia los Satyrinae y Libytheinae, consideradas hasta hace unos años con nivel familia (Heikkilä et al., 2012)).

El orden Lepidoptera, y en particular las mariposas diurnas, es uno de los grupos de insectos mejor estudiados (Dennis et al., 2006). Debido al buen nivel de conocimiento que se tiene de la distribución geográfica de sus especies y a la sensibilidad que presentan ante los cambios ambientales, las especies de este grupo son consideradas como buenos bioindicadores del estado de conservación de los ecosistemas y de los efectos del cambio climático (Stefanescu et al. 2003, Romo et al., 2013, Zografou et al. 2014). Muchas de sus especies tienen unos requerimientos biológicos muy específicos que les hacen muy sensibles a las alteraciones de los hábitats. En consecuencia, el proceso de cambio climático, unido a la creciente fragmentación y destrucción de hábitats y a la intensificación de la agricultura (Nilsson et al., 2013), están provocando cambios en la distribución espacial de sus especies y un aislamiento creciente de sus poblaciones (Franklin et al., 2002). Por ello el análisis de la presencia o ausencia de especies de mariposas diurnas en los ecosistemas, puede ser utilizado como un buen indicador del estado de conservación de los mismos (Andrade-C, 1998; Bethzholtz et al., 2013).

Durante las últimas décadas, en el mundo rural se han llevado a cabo profundos cambios de usos del suelo y de sistemas productivos, con un abandono creciente de sistemas tradicionales de gestión agrosilvopastoral y la sustitución de pequeñas unidades productivas por grandes extensiones de monocultivos intensivos que afecta a la heterogeneidad de hábitats a nivel paisaje y a la riqueza de su biodiversidad (Batáry et al. 2015). Muchos de los hábitats de las mariposas se han visto reducidos o incluso eliminados, lo que ha supuesto un factor negativo para la supervivencia de sus poblaciones que además se han visto afectadas por la aplicación de elevadas dosis de agroquímicos y productos fitosanitarios en las áreas de nuevos tipos de producción agrícola (Gilbum et al.2015). Por otra parte, la creciente disminución de la presencia de ganado y el abandono de la actividad de pastoreo en amplias zonas de Espańa y resto de Europa, ha propiciado la aparición de procesos de sustitución de especies vegetales provocando cambios en la composición de las comunidades de mariposas (Nilsson et al., 2013).

Por todo ello, el conocimiento de las especies de lepidópteros en un área determinada es una valiosa herramienta que permite evaluar su estado de conservación. De este modo, mediante el estudio de las 
comunidades de este grupo de insectos, se puede contribuir de manera eficaz a los programas de conservación y gestión del medio natural, como ha sido ampliamente puesto de manifiesto por diversas organizaciones como Butterfly Conservation Europe - BCE (http://www.bc-europe.eu/), North American Butterfly Association - NABA (http://www. naba.org/) o la Asociación Española para la Protección de las Mariposas y su Medio - ZERYNTHIA (http://www.asociacion-zerynthia.org/), surgida en los últimos años.

En este estudio se ha planteado conocer las poblaciones de mariposas diurnas de la Estación Biológica de Torretes, perteneciente al Ayuntamiento de IBI y cedido en custodia del territorio al Centro Iberoamericano de la Biodiversidad (CIBIO), instituto universitario de investigación de la Universidad de Alicante. Es un espacio protegido de 53 ha de monte mediterráneo que alberga un Jardín etnobotánico y área de cultivos mediterráneos.

En este espacio se llevan a cabo numerosos trabajos de conservación y mejora de hábitats y se desarrollan numerosos programas de investigación y conservación de biodiversidad mediterránea (Ríos \& Martínez-Francés, 2007). En el marco de estos programas se están llevando a cabo estudios sobre la diversidad y biología de los insectos (Sánchez et al., 2017; Palomo et al., 2017), no existiendo datos sobre las mariposas diurnas a pesar de ser uno de los grupos mejor conocidos de la provincia Alicante (Robert et al., 1983; Huemer y Wieser, 2006). Por este motivo se planteó estudiar la diversidad y abundancia de este grupo de insectos y los requerimientos de hábitats de sus especies, todo ello con el fin de poder contribuir a la conservación de sus poblaciones.

\section{MATERIAL Y MÉTODOS}

El trabajo de campo, como se ha indicado, ha sido realizado en la Estación Biológica de Torretes, situado dentro del término municipal de Ibi, al norte de la provincia de Alicante. Se sitúa entre los 900 y los 1.100 metros de altitud y está ubicado en la cara sur del Parque Natural del Carrascal de la Font Roja, entre los $30^{\circ}$ y $40^{\circ}$ de latitud.
Del total de sus 53 h, 44 de ellas corresponden a superficie forestal y el resto a superficie agraria. La sequía estival dura aproximadamente tres meses y las heladas se producen durante los meses invernales (Ríos \& Martínez-Francés, 2007). Sus precipitaciones se encuentran entre los 250 y los $1.500 \mathrm{~mm}$, son estacionalmente irregulares y con frecuencia se dan fenómenos de torrencialidad (Sánchez et al., 2017).

El material estudiado se registró en el periodo de tiempo comprendido entre mayo de 2016 y junio de 2017. También se ha incluido material recolectado por Esteban Sánchez y Nuria Aniorte con trampa Malaise, entre mayo de 2015 y abril de 2016, en un estudio sobre la diversidad de sírfidos (Diptera: Syrphidae) de este espacio (Sánchez et al., 2017).

El método de muestreo consistió en el uso de la manga entomológica, identificándose in situ las especies y siendo liberados a continuación los ejemplares. Solo en caso de duda para su correcta identificación, se llevó el ejemplar al laboratorio (Abelló et al., 2004). Para el mangueo se diseñaron 6 transectos de aproximadamente 200 metros de longitud, atendiendo a la diversidad de hábitats. Los muestreos se realizaron cada 2 semanas a lo largo del periodo de un año, y los transectos se recorrieron siguiendo la metodología propuesta por van Swaay et al. (2012), contabilizándose todos los ejemplares dentro del espacio situado a $2,5 \mathrm{~m}$ a cada lado y 5 $\mathrm{m}$ por delante y por encima del observador.

De acuerdo con la representatividad de hábitats en la Estación Biológica de Torretes (Ríos \& Martínez-Francés, 2007; Ríos comunicación en persona) se consideraron los siguientes transectos:

T1. Barranco 1: Espacio que forma parte del barranco de San Pascual. Es una zona poco antropizada, apenas expuesta a las transformaciones y en la que existe una humedad mayor y temperaturas más suaves. En el extremo norte existe agua embalsada de manera permanente. En cuanto a la vegetación existente destacan asociaciones vegetales como Hedero hecilis-Quercetum rotundifoliae Costa, Peris \& Stübing 1987, Fraxinus orni-Qurcetum faginea y Jasonio glutinosae-Teucreitum thymifolli Rigual, Esteve \& Rivas Goday 1963 corr. Alcaráz \& De la Torre 1988, correspondientes a la vegetación de roquedo. En el punto de agua se encuentran formaciones de juncales churreros, pertenecientes a la asociación 
Holoschoenetum vulgaris Br.-Bl. Ex Tchou 1948.

T2. Barranco 2: Este espacio está más antropizado, con grava en el camino y en una de las laderas con vegetación del Jardín Etnobotánico. Este transecto termina en la balsa de T1 y posee en su extremo una retención adicional de agua. Destaca la asociación vegetal Teucrio homotrichi-Ulicetum parviflori Alcaráz \& De la Torre 1988 corr. RivasMartínez \& al. 2002, junto a varias especies plantadas como Fraxinus ornus L., Arbutus unedo L., Acer granatense Boiss, Prunus mahaleb L., Ruscus aculeatus L. o Rhamnus alaternus L. En el punto de agua más septentrional, encontramos Iris pseudacorus L.

T3. Camino Central: Siguiendo el trayecto del camino principal de la Estación, este transecto posee escasa cobertura vegetal y la vegetación difiere a lo largo del mismo, encontrando la asociación Teucrio pseudochamaepityos-Brachypodietum ramosi $\mathrm{O}$. Bolòs 1957, pasando por una rosaleda y culminando con una plantación de Liliáceas. Posee pequeños puntos de agua a lo largo del mismo.

T4. Pinar: Situado en el sudoeste de la Estación, corresponde a parte del Jardín Etnobotánico. En su tramo inicial encontramos colecciones de Asteráceas, Rutáceas y Lamiáceas. Dentro del pinar, se reconoce principalmente la asociación Teucrio pseudochamaepityos-Brachypodietum ramosi. La cobertura vegetal es muy alta casi en la totalidad del transecto.

T5. Salvietum: Con su inicio en el rincón del Jardín Etnobotánico dedicado a las plantas comestibles, este transecto se caracteriza por una alta diversidad de especies de Salvia, como Salvia heerii Regel, Salvia coccinea Juss. Ex Murr., Salvia darcyi J. Compton o Salvia greggi Gray. La cobertura vegetal sobre este transecto es muy escasa.

T6. Camino a Font Roja: Fuera de los límites del Jardín Etnobotánico, en el camino central que conecta con el Parque Natural de la Font Roja, ubicado en la ladera de la Sierra del Menetjador. Este transecto se encuentra al abrigo del viento y tiene poca cobertura vegetal. La vegetación principal corresponde a un espartal, predominando la asociación Helictotricho filifolii-Stipetum tenacissimae Costa, Peris \& Stübing 1989.

Para la identificación de las especies se han tenido en cuenta principalmente las obras de Robert (1983) y Fernández-Rubio (1991), así como otra bibliografía especializada y la colección de lepidópteros de la colección CEUA del instituto CIBIO.

El material recolectado se ha depositado en la Colección Entomológica de la Universidad de Alicante (CEUA), depositada y custodiada en el instituto CIBIO de la Universidad de Alicante.

\section{RESULTADOS}

Se han registrado un total de 563 individuos, pertenecientes a 32 especies de Lepidópteros Ropalóceros (Tabla 1).

En los gráficos 1 y 2 se pueden observar la abundancia y riqueza de especies en cada transecto.

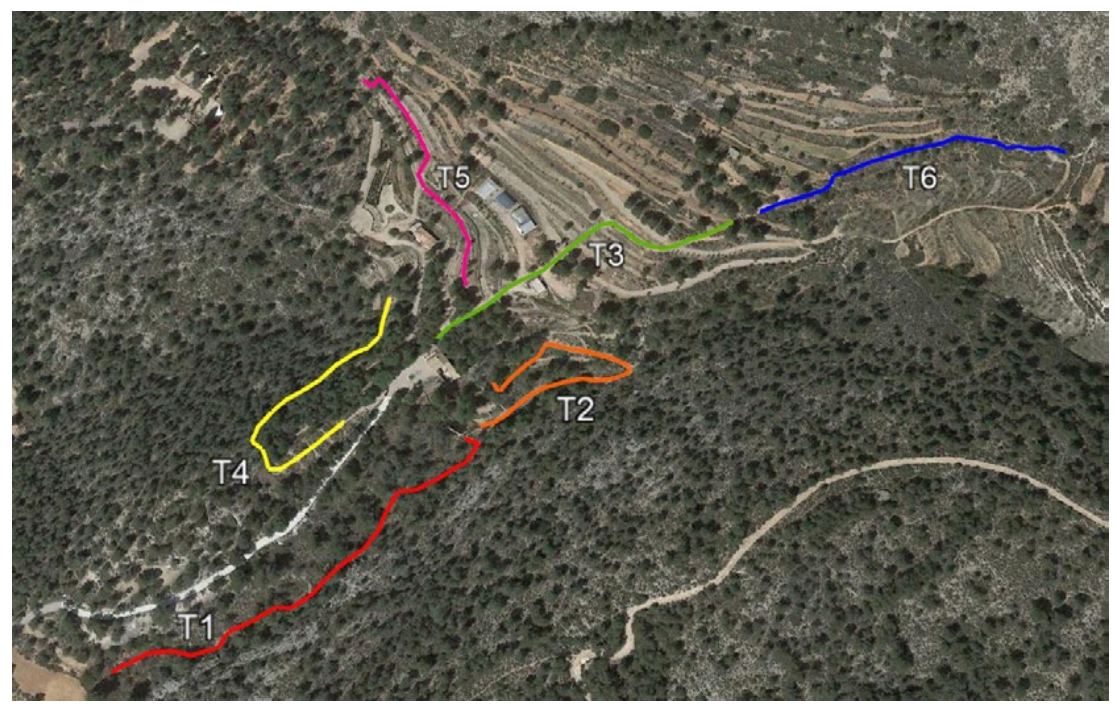

Figura 1. Ubicación de los transectos de seguimiento de mariposas diurnas. Leyenda: T1, Barranco 1.T2, Barranco 2. T3, Camino Central. T4, Pinar. T5, Salvietum. T6, Camino a Font Roja. 


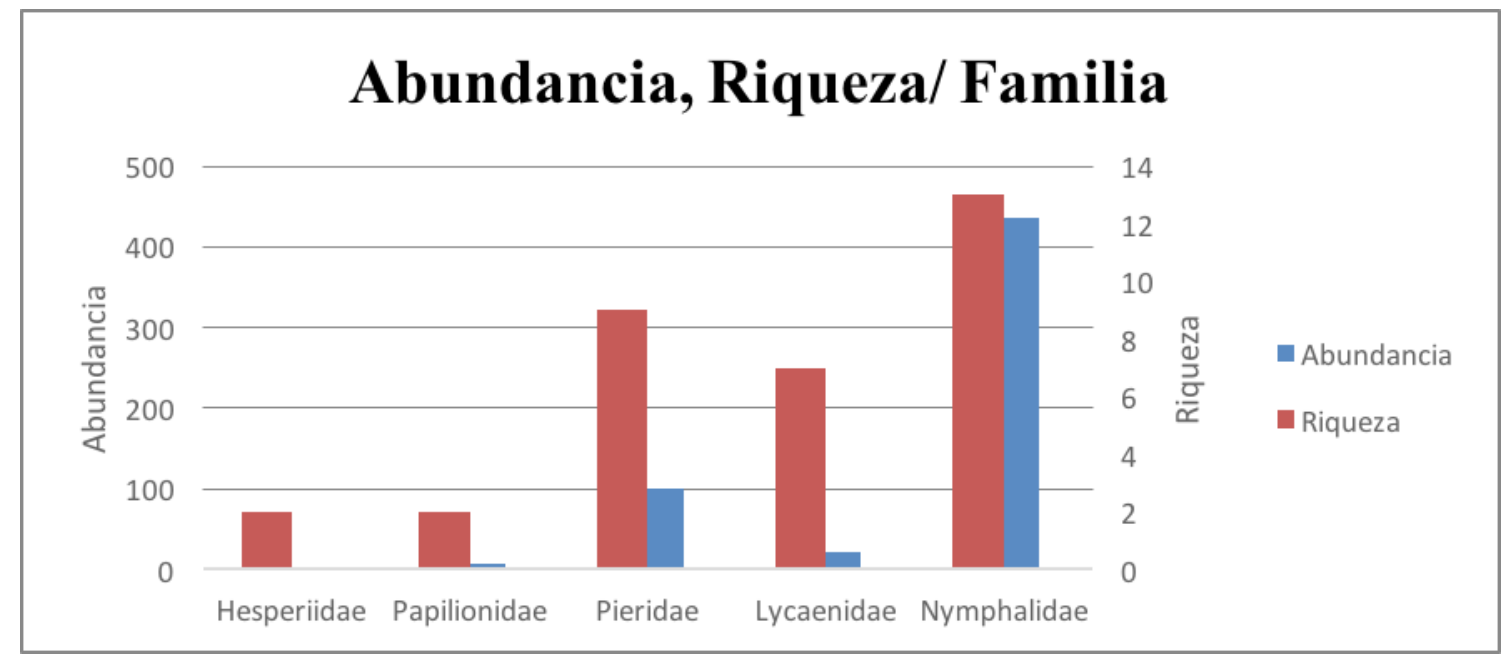

Gráfico 3. Composición (Abundancia y riqueza) de las familias incluidas en el estudio.

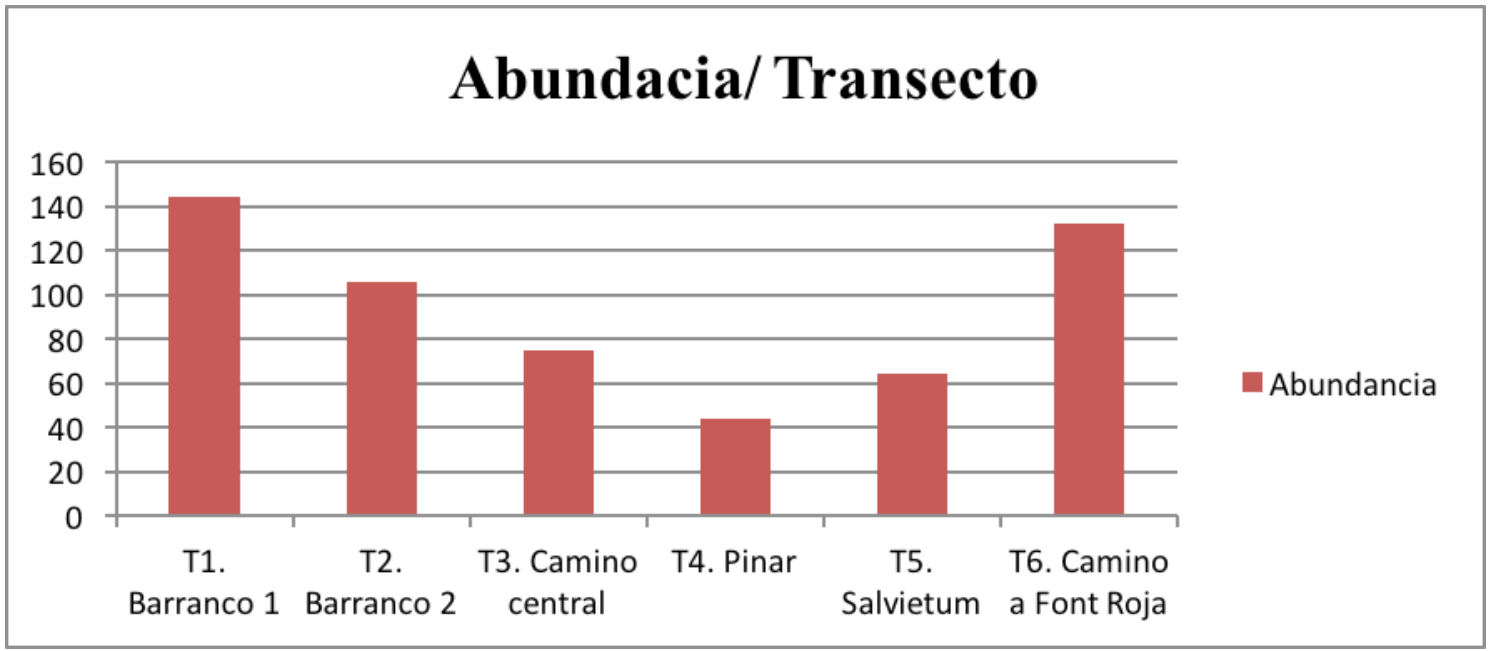

Gráfico 1. Relación entre la abundancia de ejemplares y los diferentes transectos.

\section{Riqueza/ Transecto}

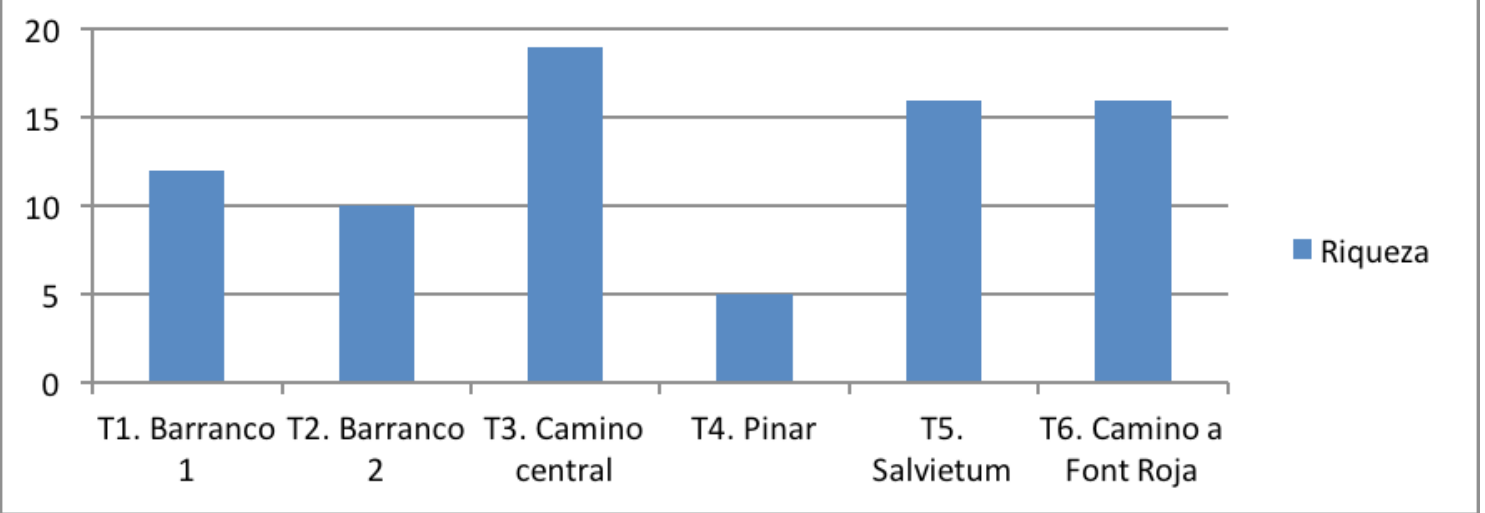

Gráfico 2. Relación entre la riqueza de especies y los diferentes transectos. 


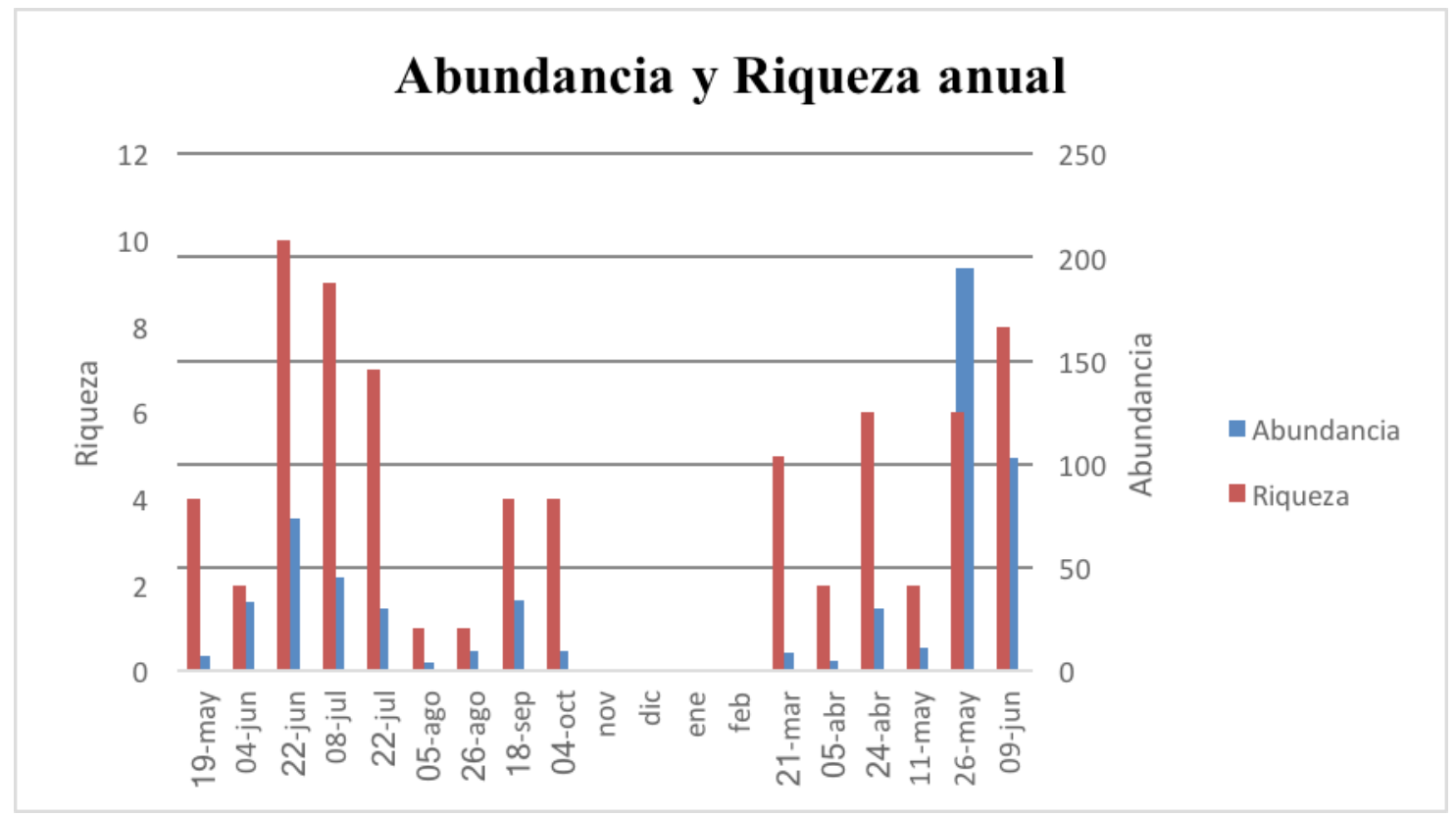

Gráfico 4. Abundancia y riqueza obtenidas durante el periodo de estudio.

\begin{tabular}{|c|c|c|c|c|c|c|c|c|c|c|c|c|c|c|c|}
\hline \multirow{2}{*}{$\begin{array}{l}\text { Media = 88,1 } \\
\text { Desviación típica = } 118\end{array}$} & \multicolumn{9}{|c|}{2016} & \multicolumn{6}{|c|}{2017} \\
\hline & $19-5$ & $4-6$ & $22-6$ & $8-7$ & $22-7$ & $5-8$ & $26-8$ & $18-9$ & $4-10$ & $21-3$ & $5-4$ & $24-4$ & $11-5$ & $26-5$ & 9-6 \\
\hline T1. Barranco 1 & 20 & 40 & 110 & 30 & 20 & - & 20 & 80 & 50 & - & 20 & - & - & 650 & 480 \\
\hline T2. $\mathrm{B}$ & - & 20 & 90 & 80 & 80 & - & 10 & 130 & 20 & - & - & 40 & - & 530 & 210 \\
\hline T3. C & 10 & 60 & 190 & 160 & 80 & - & 20 & 30 & 10 & 20 & - & 120 & 10 & 90 & 80 \\
\hline T4. Pinar & - & 70 & 40 & 20 & 30 & - & - & - & - & - & - & - & 20 & 190 & 40 \\
\hline T5. Salvietum & 20 & 60 & 100 & 70 & 30 & - & 30 & 100 & 20 & 30 & 10 & 40 & 10 & 140 & 60 \\
\hline T6. Camino a Font Roja & 20 & 80 & 200 & 90 & 60 & 40 & 20 & - & - & 40 & 20 & 100 & 70 & 350 & 160 \\
\hline
\end{tabular}

Tabla 1. Densidad, expresada en ind/ha, registrada en cada transecto durante todo el periodo de estudio. En recuadro en rojo se muestran las de mayor abundacia.

Para calcular la densidad (ind/ ha) se han tenido en cuenta la longitud de los transectos, (aproximadamente 200 metros) y el número de individuos registrados en un recuadrado imaginario de 5 metros de ancho $(200 \times 5=1000 \mathrm{~m} 2=0,1$ ha $)$. Se ha calculado la media y la desviación típica para el conjunto.

\section{DISCUSIÓN}

Se han citado en el Parque Natural de la Font Roja 74 especies de mariposas dirunas (Cantó et al., 2017), de las cuales 32 se han localizado en el espacio de la Estación Biológica de Torretes. Es un número de especies elevado si tenemos en cuenta que la superficie del carrascal protegido de la Font Roja es de 2.298 ha y presenta una alta diversidad de hábitats, mientras que Torretes es una antigua finca de secano de 53 ha que está situada en la solana de este parque natural.

Del conjunto de especies encontradas, destaca el alto número de ejemplares (282 individuos) registrados de Pyronia bathseba (Fabricius, 1793), especie generalmente muy abundante en laderas, 
Tabla 2. Listado de especies de mariposas diurnas registradas. Los números en las celdas centrales representan los transectos donde fueron registradas. El total indica la abundancia de dicha especie. Las especies con un asterisco han sido capturadas a través de trampa Malaise. Leyenda: 1, Barranco 1; 2, Barranco 2; 3, Camino Central; 4, Pinar; 5, Salvietum; 6, Camino a Font Roja.

\begin{tabular}{|c|c|c|c|c|c|c|c|c|c|c|c|c|c|c|c|c|}
\hline & \multicolumn{9}{|c|}{2016} & \multicolumn{6}{|c|}{2017} & \multirow{2}{*}{ Total } \\
\hline & $19-5$ & $4-6$ & $22-6$ & 8-7 & $22-7$ & $5-8$ & $26-8$ & $18-9$ & $4-10$ & $21-3$ & $5-4$ & $24-4$ & $11-5$ & $26-5$ & $9-6$ & \\
\hline Fam. Hesperiidae & \#\#\# & \#\#\# & \#\#\# & \#\#\# & \#\#\# & \#\#\# & \#\#\# & \#\#\# & \#\#\# & \#\#\# & \#\#\# & \#\#\# & \#\#\# & \#\#\# & \#\#\# & \#\#\# \\
\hline $\begin{array}{l}\text { Erynnis tages } \\
\text { (Linnaeus, 1758)* }\end{array}$ & & & & & & & & & & & & & & & & 1 \\
\hline $\begin{array}{l}\text { Muschampia proto } \\
\text { (Ochsenheimer, } \\
\text { 1808)* }\end{array}$ & & & & & & & & & & & & & & & & 1 \\
\hline Fam. Papilionidae & \#\#\# & \#\#\# & \#\#\# & \#\#\# & \#\#\# & \#\#\# & $\# \#$ & \#\#\# & \#\#\# & \#\#\# & \#\#\# & \#\#\# & $\# \#$ & \#\#\# & \#\#\# & $\# \#$ \\
\hline $\begin{array}{l}\text { Iphiclides feisthamelii } \\
\text { (Duponchel, 1832) }\end{array}$ & & & 3 & & 3,6 & & & & & 5,6 & & & & & & 5 \\
\hline $\begin{array}{l}\text { Papilio machaon } \\
\text { (Linnaeus, 1758) }\end{array}$ & & & & & 5 & & & 5 & & & & & & & & 2 \\
\hline Fam. Pieridae & $\# \#$ & \#\#\# & $\# \#$ & \#\#\# & \#\#\# & \#\#\# & $\# \#$ & \#\#\# & $\# \#$ & $\# \#$ & \#\#\# & \#\#\# & $\# \#$ & $\# \# \#$ & $\# \#$ & \#\# \\
\hline $\begin{array}{l}\text { Anthocharis } \\
\text { euphenoides } \\
\text { (Staudinger, 1869) }\end{array}$ & & & & & & & & & & 3,6 & & $\begin{array}{r}2,3 \\
5,6\end{array}$ & & & & 7 \\
\hline $\begin{array}{l}\text { Aporia crataegi } \\
\text { (Linnaeus. 1758)* }\end{array}$ & & & & & & & & & & & & & & & & 1 \\
\hline $\begin{array}{l}\text { Colias crocea } \\
\text { (Fourcroy, 1758) }\end{array}$ & & & & & & & & & & & & & & & 3 & 1 \\
\hline $\begin{array}{l}\text { Euchloe crameri } \\
\text { (Butler, 1869) }\end{array}$ & $\begin{array}{c}1,3 \\
6\end{array}$ & 3,6 & & & & & & & & 5 & & & 3 & & & 7 \\
\hline $\begin{array}{l}\text { Gonepteryx cleopatra } \\
\text { (Linnaeus, 1767) }\end{array}$ & & & & & & & & & & & & & & 2,3 & 3,5 & 4 \\
\hline $\begin{array}{l}\text { Gonepteryx rhamni } \\
\text { (Linnaeus, 1758) }\end{array}$ & & & 3 & & & & & & & & & & & & & 4 \\
\hline $\begin{array}{l}\text { Pieris mannii (Mayer, } \\
\text { 1851) }\end{array}$ & & & $\begin{array}{l}1,2, \\
5,6\end{array}$ & 2 & & & & & & 3,6 & & $\begin{array}{r}2,3 \\
5,6\end{array}$ & & $\begin{array}{c}1,2, \\
5\end{array}$ & $\begin{array}{c}1-3 \\
6\end{array}$ & 60 \\
\hline $\begin{array}{l}\text { Pieris rapae (Linnaeus, } \\
\text { 1758) }\end{array}$ & & & & & 4 & & & & & & & $\begin{array}{r}2,3, \\
5,6\end{array}$ & & & & 10 \\
\hline $\begin{array}{l}\text { Pontia daplidice } \\
\text { (Linnaeus, 1758) }\end{array}$ & & & & & & & & & & & & & & $\begin{array}{c}1,3, \\
6\end{array}$ & & 6 \\
\hline Fam. Lycaenidae & \#\#\# & \#\#\# & \#\#\# & \#\#\# & \#\#\# & $\# \#$ & \#\#\# & \#\#\# & \#\#\# & $\# \#$ & \#\#\# & \#\#\# & $\# \#$ & \#\#\# & $\# \# \#$ & \#\#\# \\
\hline $\begin{array}{l}\text { Aricia cramera } \\
\text { (Eschscholtz, 1821) }\end{array}$ & & & & & & & & & & & & & & 2,6 & & 2 \\
\hline $\begin{array}{l}\text { Callophrys rubi } \\
\text { (Linnaeus, 1758) }\end{array}$ & & & & & & & & & & 5,6 & $\begin{array}{c}1,5, \\
6\end{array}$ & & & & & 5 \\
\hline $\begin{array}{l}\text { Lampides boeticus } \\
\text { (Linnaeus, 1767)* }\end{array}$ & & & & & & & & & & & & & & & & 1 \\
\hline $\begin{array}{l}\text { Philotes panoptes } \\
\text { (Huebner, 1813)* }\end{array}$ & & & & & & & & & & & & & & & & 1 \\
\hline
\end{tabular}




\begin{tabular}{|c|c|c|c|c|c|c|c|c|c|c|c|c|c|c|c|c|}
\hline $\begin{array}{l}\text { Polyommatus } \\
\text { bellargus } \\
\text { (Rottemburg, 1775) }\end{array}$ & & & & 6 & & & & & & & & 2,3 & & & & 3 \\
\hline $\begin{array}{l}\text { Polyommatus icarus } \\
\text { (Rottemburg, 1775) }\end{array}$ & 5 & & 3 & & & & & & 5 & & & & & & & 4 \\
\hline $\begin{array}{l}\text { Satyrium spini (Denis } \\
\text { and Schiffermueller, } \\
\text { 1775) }\end{array}$ & & & $\begin{array}{l}1,5 \\
6\end{array}$ & 3 & & & & & & & & & & & & 5 \\
\hline Fam. Nymphalidae & \#\#\# & \#\#\# & \#\#\# & $\# \#$ & \#\#\# & \#\#\# & $\# \#$ & \#\#\# & \#\#\# & \#\#\# & \#\#\# & \#\#\# & \#\#\# & \#\#\# & \#\#\# & \#\#\# \\
\hline Subfam. Lybytheinae & \#\#\# & \#\#\# & \#\#\# & $\# \#$ & $\# \#$ & \#\#\# & \#\#\# & $\# \#$ & \#\#\# & \#\#\# & \#\#\# & \#\#\# & \#\#\# & \#\#\# & \#\#\# & \#\#\# \\
\hline $\begin{array}{l}\text { Libythea celtis } \\
\text { (Laicharting, 1872)* }\end{array}$ & & & & & & & & & & & & & & & & 1 \\
\hline Subfam. Nymphalinae & \#\#\# & \#\#\# & \#\#\# & \#\#\# & \#\#\# & \#\#\# & \#\#\# & \#\#\# & \#\#\# & \#\#\# & \#\#\# & \#\#\# & \#\#\# & \#\#\# & \#\#\# & \#\#\# \\
\hline $\begin{array}{l}\text { Meliatea deione } \\
\text { (Geyer, 1883) }\end{array}$ & 5 & & & & & & & & & & & & & & & 1 \\
\hline $\begin{array}{l}\text { Vanessa atalanta } \\
\text { (Linnaeus, 1758) }\end{array}$ & & & & & & & & & 1 & & & 3 & & & & 2 \\
\hline $\begin{array}{l}\text { Vanessa cardui } \\
\text { (Linnaeus, 1758) }\end{array}$ & & & & 3,5 & 3 & & & 5 & 1,2 & & 6 & 6 & & & & 8 \\
\hline Subfam. Satyrinae & \#\#\# & \#\#\# & \#\#\# & \#\#\# & \#\#\# & \#\#\# & \#\#\# & \#\#\# & \#\#\# & \#\#\# & \#\#\# & \#\#\# & \#\#\# & \#\#\# & \#\#\# & \#\#\# \\
\hline $\begin{array}{l}\text { Coenonympha dorus } \\
\text { (Esper, 1782) }\end{array}$ & & & 3,6 & $\begin{array}{c}1,3 \\
6\end{array}$ & 1,3 & & & & & & & & & 6 & $\begin{array}{l}1,2 \\
4,6 \\
\end{array}$ & 58 \\
\hline $\begin{array}{l}\text { Hipparchia alcyone } \\
\text { (Schiffermueller, 1775) }\end{array}$ & & & $\begin{array}{l}2,3 \\
5,6\end{array}$ & $\begin{array}{c}2,5 \\
6\end{array}$ & $\begin{array}{l}1,3 \\
4,6\end{array}$ & & & & & & & & & & $\begin{array}{c}1,2, \\
6\end{array}$ & 19 \\
\hline $\begin{array}{l}\text { Hipparchia fidia } \\
\text { (Linnaeus, 1767) }\end{array}$ & & & & 5,6 & $\begin{array}{l}2,3 \\
5,6\end{array}$ & 6 & $\begin{array}{l}1-3 \\
5,6\end{array}$ & $\begin{array}{c}1,3 \\
5\end{array}$ & $\begin{array}{c}1-3, \\
5\end{array}$ & & & & & & & 48 \\
\hline $\begin{array}{l}\text { Hipparchia semele } \\
\text { (Linnaeus, 1758) }\end{array}$ & & & & & & & & & & & & & & & 1 & 1 \\
\hline $\begin{array}{l}\text { Lasiommata megera } \\
\text { (Linnaeus, 1767) }\end{array}$ & & & T3 & & & & & & & & & & & & & 3 \\
\hline $\begin{array}{l}\text { Maniola jurtina } \\
\text { (Linnaeus, 1758)* }\end{array}$ & & & & & & & & & & & & & & & & 1 \\
\hline $\begin{array}{l}\text { Melanargia occitania } \\
\text { (Esper, 1793) }\end{array}$ & & & & & & & & & & & & & & & 6 & 1 \\
\hline $\begin{array}{l}\text { Pyronia bathseba } \\
\text { (Fabricius, 1793) }\end{array}$ & 1,6 & $1-6$ & $1-6$ & $\begin{array}{c}1-4, \\
6\end{array}$ & & & & 1,2 & & & & & 4-6 & $1-6$ & $1-6$ & 282 \\
\hline $\begin{array}{l}\text { Satyrus actaea (Esper, } \\
1780 \text { ) }\end{array}$ & & & $\begin{array}{l}1-3 \\
5,6\end{array}$ & 2,3 & & & & & & & & & & & & 12 \\
\hline
\end{tabular}


zonas abruptas y arbustivas (Córdoba et al. 2006), aunque también podemos encontrarla en hábitats abiertos, con predominio de plantas herbáceas (Robert et al., 1983).

Asimismo otras tres especies presentaron un alto número de individuos, Pieris mannii (Mayer, 1851) 60 individuos, Coenonympha dorus (Esper, 1782) 58 individuos y Hipparchia fidia (Linneo, 1767) 48 individuos. Su presencia está relacionada con la existencia de hábitats con abundancia de las plantas nutricias de sus orugas, que favorecen su presencia y desarrollo (Stevens, 2004). Las orugas de P. mannii se alimentan de crucíferas, mientras las gramíneas, tales como Brachypodium retusum (Pers.) P. Beauv. 1812, constituyen el recurso trófico de las otras dos especies.

En cuanto a la información proporcionada por los resultados de diversidad y abundancia en los diferentes transectos, son el T3 (Camino central), T5 (Salvietum) y T6 (Camino a Font Roja) los que presentan una mayor abundancia y riqueza, mostrando 16, 16 y 19 especies respectivamente. Esto se debe al carácter abierto de estos espacios, con cobertura vegetal herbácea, y sometidos a una mayor insolación. Además sus hábitats están en cierta manera protegidos del viento, disminuyendo de este modo la perdida de calor por corrientes de aire y permitiendo el vuelo de los ejemplares (Barker \& Herman, 1976).

Las mariposas diurnas se termorregulan mediante exposición al sol y reposo sobre sustratos calientes por la radiación solar (Roland, 1982). Es probablemente por esta razón que la abundancia y riqueza más baja sea en el transecto T4 (Pinar) caracterizado por elevada cobertura vegetal y con menor insolación a nivel de sustrato.

Cabe destacar asimismo el elevado número de individuos de $P$. bathseba registrados en los transectos T1 (Barranco 1) y T2 (Barranco 2) con 70 y 40 individuos respectivamente. Este hecho puede deberse al carácter oportunista de esta especie, así como a la presencia de charcas de agua en ambos transectos, lo que contribuye de forma positiva a la presencia de imagos de esta especie (Niell, 2007; Ruchi et al., 2012)

La diferencia entre el número de especies registradas en este estudio (32 especies), frente a los datos aportados por Robert et al. (1983) y Viejo et al. (1994) (48 especies) en esta zona, es probable que se deba al efecto de los recientes cambios ambientales ocurridos en estos últimos 30 ańos y al incremento considerable de las temperaturas medias anuales (Cruz \& Martínez, 2015). Los datos aportados por la Agencia Estatal de Meteorología (www.aemet. es) en relación al periodo de años comprendido entre 2010 y 2017, indican que las temperaturas fueron más elevadas que en años precedentes y las precipitaciones muy escasas, situación que repercute sobre las comunidades de lepidópteros que presen$\tan$ en consecuencia menor riqueza y abundancia (Robinson et al., 2012). Los periodos de sequía prolongados afectan negativamente al desarrollo larvario, pudiendo llegar a provocar la muerte de las orugas (Descimon et al., 2005). Asimismo las altas temperaturas conllevan procesos de mayor evaporación y en consecuencia mayor nubosidad (Matter et al., 2011) influyendo negativamente en los procesos de termorregulación y por tanto en la actividad y procesos de dispersión de los imagos (Gilbert \& Singer, 1975).

Estos primeros resultados sobre mariposas diurnas de la Estación Biológica/ Jardín Botánico de Torretes constituyen un nuevo soporte para la conservación de este espacio (Sánchez et al., 2017; Palomo et al., 2017) y su posible reconocimiento como futura Reserva Entomológica de acuerdo con las directrices de la Asociación española de Entomología (www.entomologica.es). El hecho de que la Estación Biológica Torretes se encuentre gestionada y protegida por el instituto de investigación $\mathrm{CIBIO}$ de la Universidad de Alicante en colaboración con el Ayuntamiento de Ibi, permite convertir este espacio en un observatorio de los efectos que sobre las poblaciones de insectos tiene el cambio global y en particular del calentamiento climático. Esta estación podría complementarse con otros puntos del Parque Natural de la Font Roja situados a más altitud y en umbría, donde se podrían llevar a cabo estudios sobre los factores que determinan la presencia y la abundancia de las especies en el monte mediterráneo del levante ibérico. Esto permitiría sentar las bases para poner en marcha programas dirigidos a la conservación de las especies de insectos, y en particular de sus hábitats. 


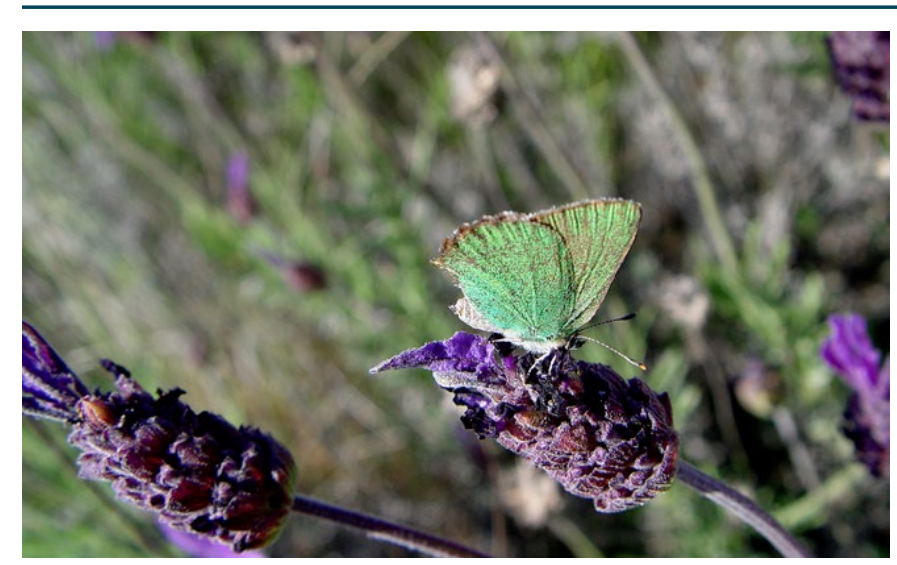

Callophris rubi. Fotografía E. Galante

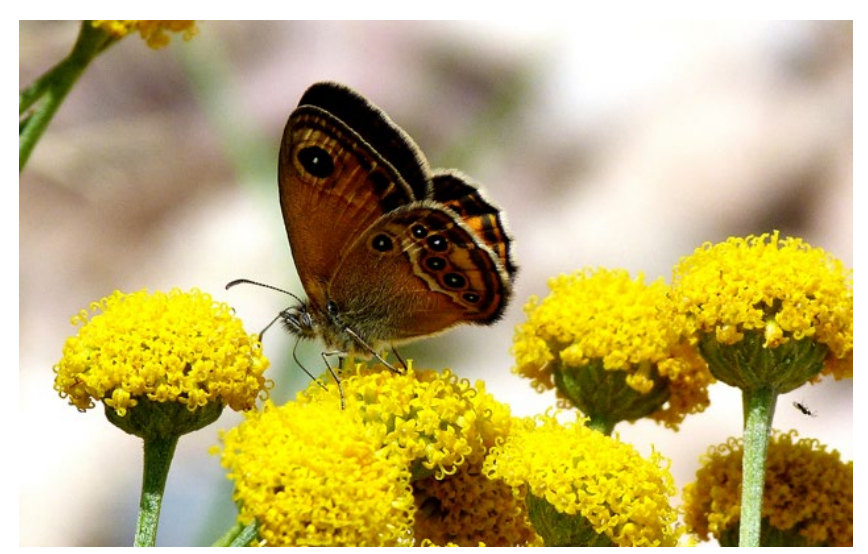

Coenonympha dorus. Fotorafía E. Galante

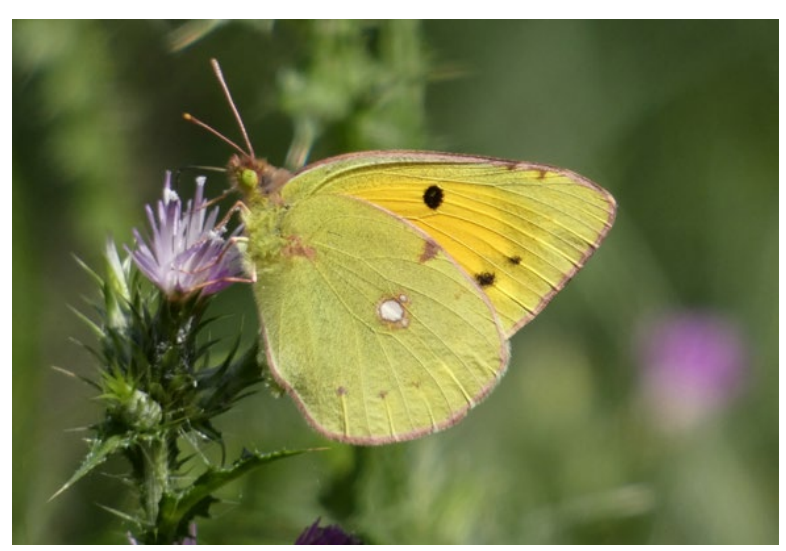

Colias croceus. Fotografia E. G alante

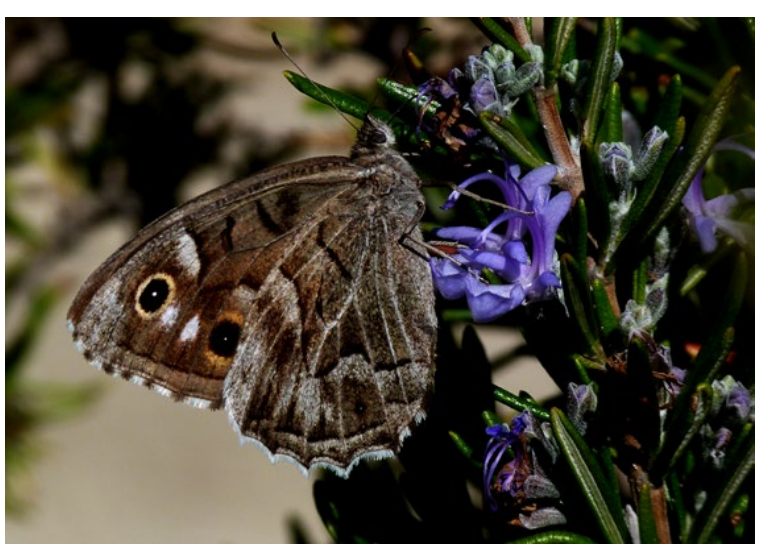

Hipparchia fidia. Fotorafía E.

Galante

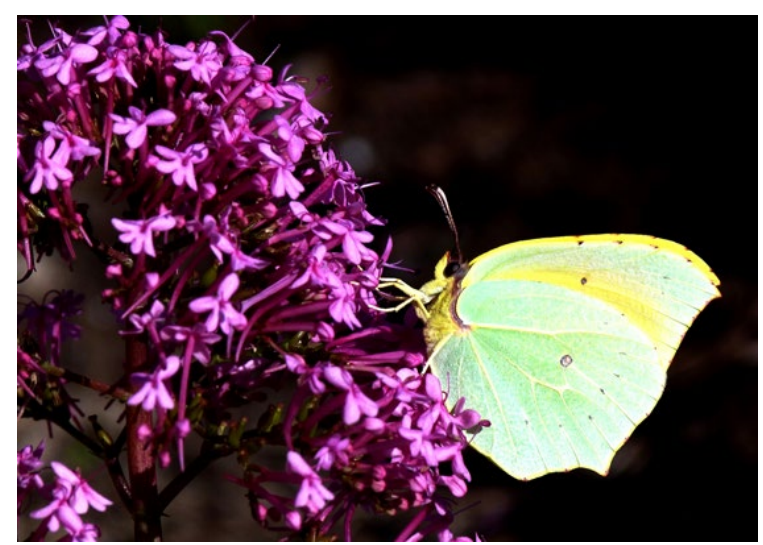

Gonepteryx cleopatra. Fotografía E. Galante

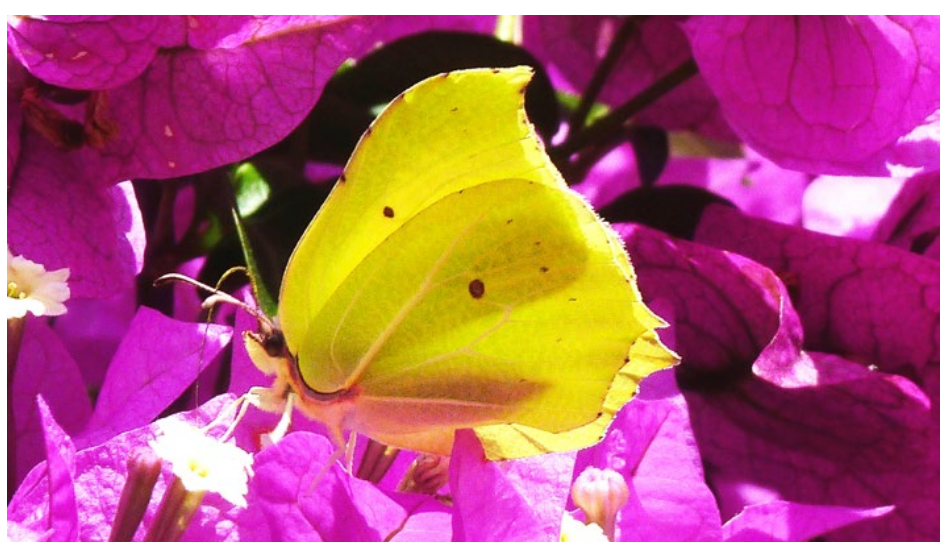

Gonepteryx rhamnii. Fotografía E. Galante 


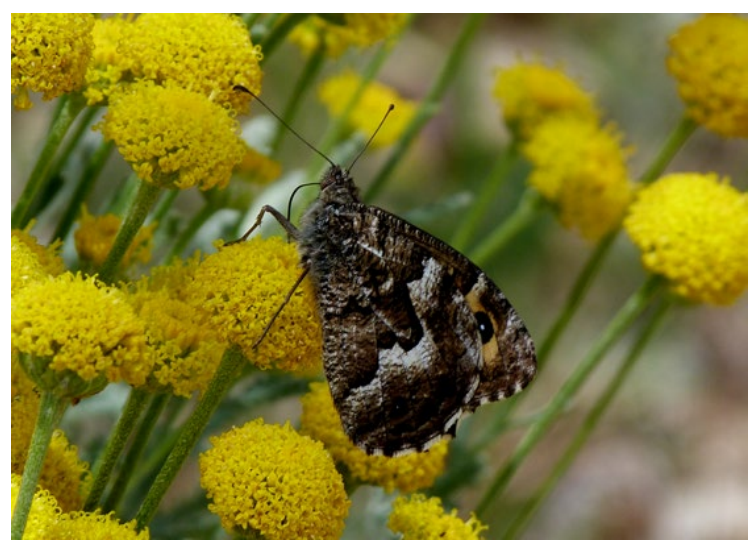

Hipparchia semele. Fotografía E. Galante

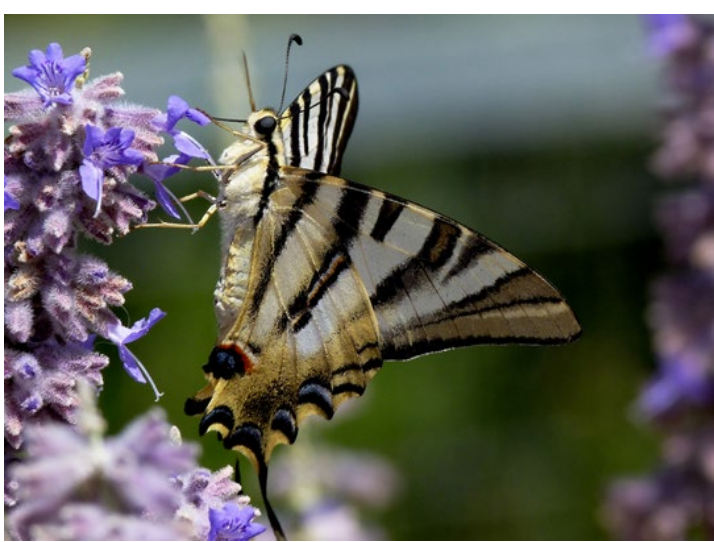

Iphiclides podalirius. Fotografía E. Galante

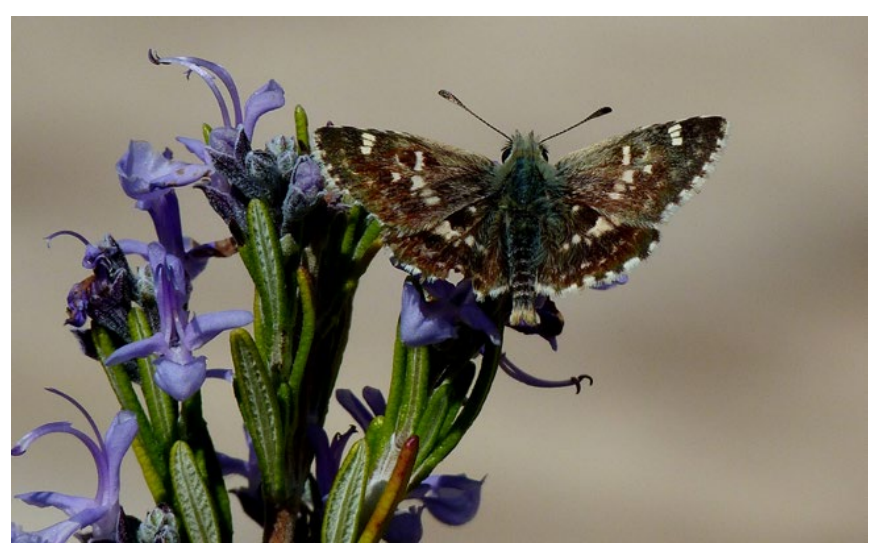

Muschampia proto. Fotografía E. Galante

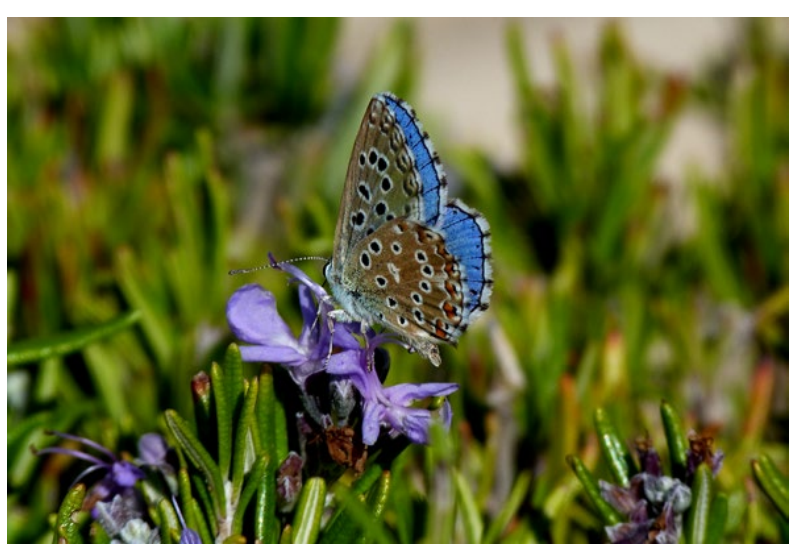

Ployommatus icarus. Fotografía E. Galante

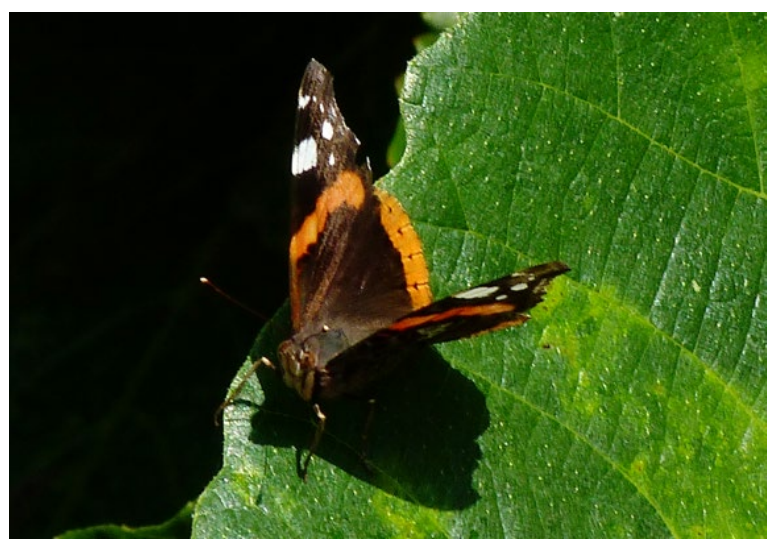

Vanessa atalanta. Fotografía E. Galante

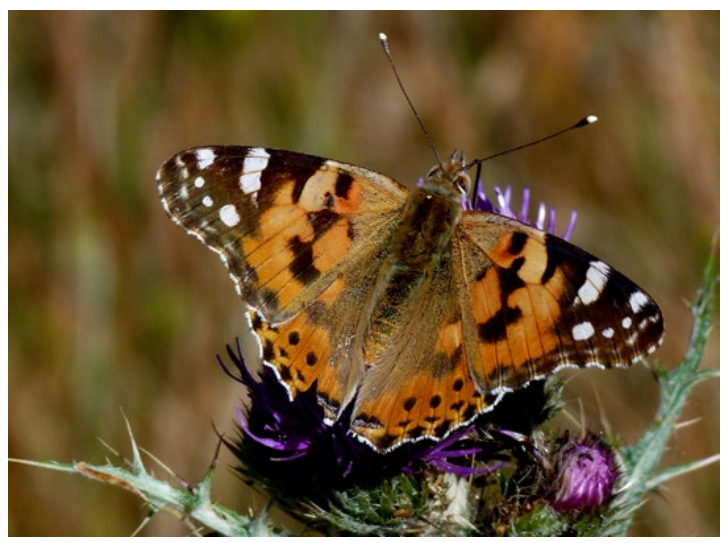

Vanessa cardui. Fotografía E. Galante 


\section{REFERENCIAS}

Abelló, P., Barrientos, J.A. (2004). Curso Práctico de Entomología. Asociación Española de Entomología, CIBIO: Centro Iberoamericano de la Biodiversidad (Alicante), Universitat Autónoma de Barcelona. Bellaterra, Alicante.

Andrade, G. (1998). Utilización de las mariposas como bioindicadoras del tipo de hábitat y su biodiversidad en Colombia. Revista de la Academia Colombiana de Ciencias Exactas, Físicas y Naturales, 22(84): 407-421.

Barker, J.F. \& Herman, W.S. (1976). Effect of photoperiod and temperature on reproduction of the monarch butterfly, Danaus plexippus. Journal of Insect Physiology, 22(12):1565-1568.

Batáry, P., Dicks, L.V., Kleijn, D., Sutherland, W.J. (2015). The role of agri-environment schemes in conservation and environmental management. Conservation Biology 29:1006-1016

Betzholtz, P. E., Pettersson, L. B., Ryrholm, N., \& Franzén, M. (2013). With that diet, you will go far: trait-based analysis reveals a link between rapid range expansion and a nitrogen-favoured diet. Proceedings of the Royal Society of London B: Biological Sciences, 280(1750):20122305.

Cantó J.L., Acosta J.A. \& Sanchís, M.J. (2017). Mariposas diurnas del Carrascal de la Font Roja (Papilionoidea y Heperoidea). Iberis 9:27-30.

Convey, P. (2004) Recent Lepidoptera records from subAntartic South Gerogia. Polar Biology, 28(2): 108-110.

Cruz, Y.Y.P., \& Martínez, P.C.C. (2015). Cambio climático: Bases científicas y escepticismo. CULCyT: Cultura Cientifica y Tecnológica, 46 (9).

Dennis, R. L. H., Shreeve, T. G., Isaac, N. J. B., Roy, D. B., Hardy, P. B., Fox, R. \& Asher, J. (2006). The effects of visual apparency on bias in butterfly recording and monitoring. Biological Conservation 128: 486-492.

Descimon, H., Bachelard, P., Boitier, E. \& Pierrat, V. (2005) Decline and extinction of Parnassius apollo populations in France - continued. En: Kühn, E., Feldmann, R., Thomas, J. A. \& Settele, J. (eds), Studies on the Ecology and Conservation of Butterflies in Europe. Vol. 1: General Concepts and Case Studies, Sofia-Moscow. pp. 114-115.
Fernández-Rubio, F. (1991). Guía de mariposas diurnas de la peninsula ibérica, Baleares, Canarias, Azores y Madeira: Libhyteidae, Nymphalidae, Riodinidae y Lycaenidae. Ediciones Pirámide. España. 408 pp.

Franklin, A. B., Noon, B. R., \& George, T. L. (2002). What is habitat fragmentation? Studies in avian biology, 25:2029.

Gilbert, L. E., \& Singer, M. C. (1975). Butterfly ecology. Annual Review of Ecology and Systematics, 6(1):365-395.

Gilburn A.S., Bunnefeld N., Wilson J.M., Brereton T.M. \& Goulson D. (2015). Are neonicotinoid insecticides driving declines of widespread butterflies? PeerJ.3: e1402

Heikkilä, M., Kaila, L., Mutanen, M., Peña, C. \& Wahlberg, N. (2012). Cretaceous origin and repeated tertiary diversification of the redefined butterflies. Proceedings of the Royal Society B: Biological Sciences, 279(1731):10931099.

Huemer, P., \& Wieser, C. (2006). Addition to the faunistics of Lepidoptera in the Comunidad Valenciana (Spain) - Part I. Boletín Sociedad Entomológica Aragonesa, 1(39):271-283.

Karsholt O. \& Nieukerken E.J. (2017). Lepidoptera Papilionoidea. Fauna Europaea version 2.6.2, http:// www.faunaeur.org.

Matter, S. F., Doyle, A., Illerbrun, K., Wheeler, J., \& Roland, J. (2011). An assessment of direct and indirect effects of climate change for populations of the Rocky Mountain Apollo butterfly (Parnassius smintheus Doubleday). Insect Science, 18(4):385-392.

Monasterio León Y. (Coord.), García-Carrillo A., Vila Ujaldón R., Vicente-Arranz J.C., Escobés Jiménez R., García-Alamá J.A., Moreno Iriondo O,, HernándezRoldán J., Parra Arjona B. , Murria Beltrán B., Antón Lázaro J. , Baquero Herce A. , Olivares Villegas J. , Barea Azcón J.M. , PérezFernández R. , Jubany Fontanillas J. , Gutiérrez-García D., Montagud Alario S., LópezIldefonso M., Fernández Bisson P.M. \& Delgado Bello J.A. (2017). Propuesta actualizada de nombres comunes en castellano para las mariposas de la península ibérica, Baleares Y Canarias (Lepidoptera: Papilionoidea). Boletín de la Sociedad Entomológica Aragonesa (S.E.A.) 60: 463-483. 
Niell, L. E. (2007). Effects of environmental factors on butterfly species in an urban setting. University of Nevada, Reno.

Nilsson, S. G., Franzén, M. \& Pettersson, L.B. (2013). Land-use changes, farm management and the decline of butterflies associated with semi-natural grasslands in southern Sweden. Nature Conservation 6:31-48.

Numa, C., van Swaay, C., Wynhoff, I., Wiemers, M., Barrios, V., Allen, D., Sayer, C., Lopéz Munguira, M., Balletto, E., Benyamini, D., Beshkov, S., Bonelli, S., Caruana, R., Dapporto, L., Franeta, F., Garcia-Pereira, P., Karacetin, E., Katbeh-Bader, A., Maes, D., Micevski, N., Miller, R., Monteiro, E., Moulai, R., Nieto, A., Pamperis, L., Peer, G., Power, A., Sasic, M., Thompson, K., Tzirkalli, E., Verovnik, R., Warren, M. \& Welch, H. (2016) The status and distribution of Mediterranean butterflies. IUCN, Málaga.

Palomo M., C. Quirce \& E. Galante, 2017. La Estación Biológica de Torretes (Ibi, Alicante): un espacio para la conservación de Odonatos. Cuadernos de Biodiversidad 53: $52-60$

Ríos, S. \& Martínez-Francés, V. (2007). El Jardín Etnobotánico de la Estación Biológica de Torretes-Font Roja, CIBIO-Universidad de Alicante. Cuadernos de biodiversidad. 23:21.

Robert, J. H., Escarré, A., García, T., \& Martínez, P. (1983). Fauna Alicantina IV. Lepidópteros Ropalóceros, sus plantas nutricias y su distribución geográfica en la provincia de Alicante. Cuadernos de la Fauna Alicantina, Instituto de Estudios Alicantinos, 2(20): 435.

Robinson, N, Armstead, S. \& Bowers, M. D. (2012) Butterfly community ecology: the influences of habitat type, weather patterns, and dominant species in a temperate ecosystem. Entomologia Experimentalis et Applicata. 145(1):50-61

Roland, J. (1982) Melanism and diel activity of alpine Colias (Lepidopter: Pieridae). Oecologia, 53:214-221

Romo, H., Sanabria, P., \& García-Barros, E. (2013). Predicción de los impactos del cambio climático en la distribución sobre las especies de Lepidoptera. El caso del género Boloria Moore, 1900 en la Península Ibérica (Lepidoptera: Nymphalidae). SHILAP Revista de lepidopterología, 41(162).

Ruchi, N., Nirjara, G. \& Sujatha, P. (2012) What determines the abundance of butterflies? - A short search. Recent Research In Science And Technology 4(11)
Sánchez E.M., Aniorte N., Ricarte A. \& Marcos-García, M.A. (2017) Diversidad de sírfidos (Diptera: Syrphidae) de la Estación Biológica de Torretes (Alicante, Espańa). Cuadernos de Biodiversidad 52:38-45

Stefanescu C., Peñuelas J. \& Filella I. (2003). Effects of climatic change on the phenology of butterflies in the northwest Mediterranean Basin. Global Change Biology 9, 1494-1506

Stevens, D. J. (2004). Pupal development temperature alters adult phenotype in the speckled wood butterfly, Pararge aegeria. Journal of Thermal Biology, 29(4):205-210.

Van Nieukerken, E. J., Kaila, L., Kitching, I.J., Kristensen, N.P., Lees, D.C., Minet, J., Mitter, C., Mutanen, M., Regier, J.C., Simonsen, T.J., Wahlberg, N., Yen, S-H., Zahiri, R., Adamski, D., Baixeras, J., Barrtsch, D., Bengtsson, B.Å., Brown, J.W., Bucheli, S.R., Davis, D.R., De Prins, J., De Prins, W., Epstein, M.E., GentiliPoole, P., Gielis, C., Hattenschwiler, P., Hausmann, A., Holloway, J.D., Kallies, A., Karsholt, O., Kawahara, A.Y., Koster, J.C., Kozlov, M.V., Lafontaine, J.D., Lamas, G., Landry, J-F., Lee, S., Nuss, M., Park, K-T., Penz, C., Rota, J., Schintlmeister, A., Schmidt, B.C., Sohn, J-C., Solis, M.A., Tarmann, G.M., Warren, A.D., Weller, S., Yakovlev, R.V., Zolotuhin, V.V. \& Zwick, A. (2011). Order Lepidoptera Linnaeus (1758). En: Zhang, Z-Q. (ed.), Animal biodiversity: An outline of higher-level classification and survey of taxonomic richness. Zootaxa, 3148. Magnolia press, New Zealand. pp. 212221.

Van Swaay, C.A.M., Brereton, T., Kirkland, P. \& Warren, M.S. (2012). Manual para el seguimiento de mariposas. Documento VS2012.010, De Vlinderstichting/Dutch Butterfly Conservation, Butterfly Conservation UK and Butterfly Conservation Europe, Wageningen.

Viejo, J.L., Llorente, J.J., Martín, J. \& Sánchez, C. (1994). Patrones de distribución de las mariposas de Alicante (Lepidoptera, Papilionoidea y Hesperioidea), Ecología (España), (8):453-458.

Zografou, K., Kati, V., Grill, A., Wilson, R.J., Tzirkalli, E., Pamperis, L.N., et al. (2014) Signals of Climate Change in Butterfly Communities in a Mediterranean Protected Area. PLoS ONE 9(1): e87245. 
Este número ha recibido una ayuda del Vicerrectorado de Investigación y Transferencia de Conocimiento de la Universidad de Alicante
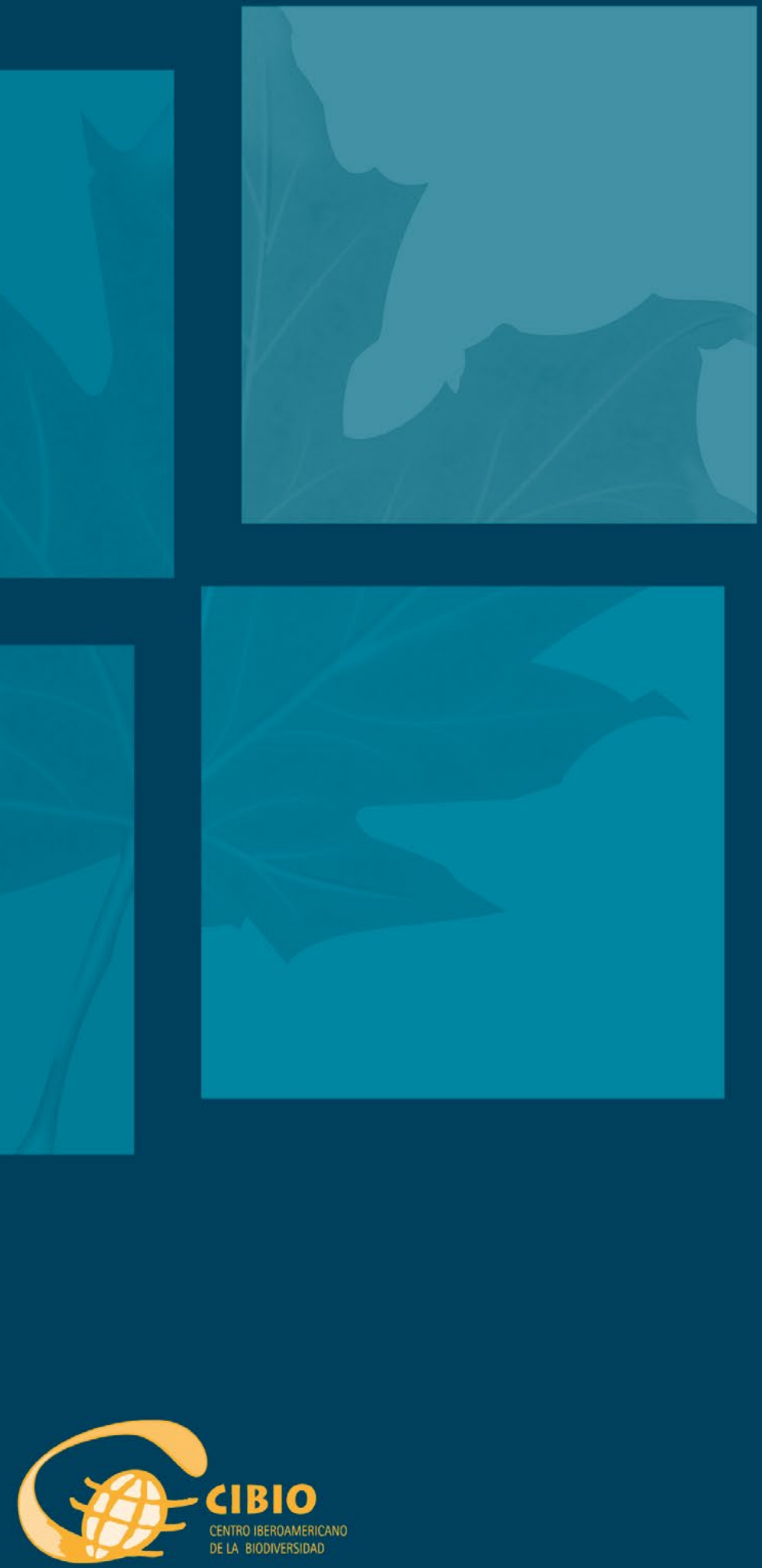

\section{Universitat d'Alacant
Universidad de Alicante}

